\title{
The LMP2 polymorphism is associated with susceptibility to acute anterior uveitis in HLA-B27 positive juvenile and adult Mexican subjects with ankylosing spondylitis
}

Walter P Maksymowych, Gian S Jhangri, Clara Gorodezky, Maria Luong, Cindy Wong, Rubén Burgos-Vargas, Monica Morenot, José Sanchez-Corona, César Ramos-Remus, Anthony S Russell

Departments of Medicine

W P Maksymowych M Luong

C Wong

A S Russell

and Public Health Sciences

G S Jhangri

University of Alberta, Canada

Rheumatology Unit, Hospital General de Mexico, Mexico City R Burgos-Vargas

Department of Immunogenetics, INDRE, SSA, Mexico City

C Gorodezky

M Morenot

Centro de

Investigacion

Biomedica, IMSS,

Guadalajara

J Sanchez-Corona

Department of

Rheumatology,

Hospital de

Especialidades CMNO,

IMSS, Guadalajara

Mexico

C Ramos-Remus

Correspondence to:

Dr W P Maksymowych, 562 Heritage Medical Research Centre, University of Alberta, Edmonton, Alberta, Canada T6G 2S2.

Accepted for publication 6 June 1997
Abstract

Introduction-An association between polymorphism of the HLA linked LMP2 locus and the development of acute anterior uveitis (AAU) has previously been described in $B 27$ positive white subjects with ankylosing spondylitis (AS). This study evaluated LMP2 alleles in two HLA-B27 positive Mexican populations of patients with spondyloarthropathy known to have a different clinical spectrum of disease from white people.

Patients and Methods-The study populations consisted of 90 AS patients from Guadalajara with predominantly adult onset disease and 80 AS patients from Mexico City with predominantly juvenile onset disease. LMP2-CfoI amplified fragment length polymorphisms were determined after polymerase chain reaction amplification and digestion with CfoI restriction enzyme.

Results-There was an increased LMP2A allelic frequency in patients who had had AAU in both Guadalajara (31.8\%) and Mexico City (33.3\%) when compared with non-AAU patients $(15.2 \%$ and $17.7 \%$ of Guadalajara and Mexico City populations, respectively). The odds ratio relating LMP2A allelic frequency and AAU for the combined population, stratified by age at onset of disease, was $2.51(p=0.01)$. LMP2 alleles did not influence the age at onset of disease or the development of peripheral arthritis.

Conclusions-These data support the view that polymorphism at the LMP2 locus is associated with the development of AAU in B27 positive subjects with AS. The requirement for both the less common LMP2 allele and HLA-B27 is consistent with the low prevalence of AAU in Mexican patients with spondyloarthritis.

(Ann Rheum Dis 1997;56:488-492)

Numerous findings derived from epidemiological studies and studies of families of patients with ankylosing spondylitis (AS) strongly implicate a role for genes additional to HLA-B27 in the pathogenesis of AS. ${ }^{12}$ Subsequent work has implicated a role for the HLA
Bw60 allele ${ }^{3}$ and polymorphism of the HLA linked proteasomal subunit LMP2 (low molecular weight polypeptide $)^{4}$ gene. ${ }^{56}$ The proteasome constitutes a large cytoplasmic multicatalytic proteinase complex implicated in antigen processing for major histocompatibility complex (MHC) class I associated antigen presentation to cytotoxic $\mathrm{T}$ cells. ${ }^{7}$ The LMP2 and LMP7 subunits of this complex are HLA encoded, $\gamma$ interferon inducible proteins, ${ }^{8}$ which influence the proteolytic specificity of the proteasome as demonstrated by transfection data ${ }^{9}$ and studies of LMP2 and LMP7 knockout mice. ${ }^{10}{ }^{11}$ The LMP2 locus encodes 2 alleles, LMP2A and LMP2B, distinguished by an arginine for histidine amino acid substitution, respectively, at position 60 of the coding sequence. ${ }^{12}$

Evaluation of the LMP2 gene polymorphism in a white population of AS patients from Edmonton, Canada, has previously shown an association between LMP2B and the occurrence of acute anterior uveitis (AAU) $)^{56}$ while a study of AS patients from England was unable to confirm this finding. ${ }^{13}$ On the other hand, a large study of LMP2 gene polymorphism in patients with predominately juvenile onset AS from Norway has not only shown an association between LMP2B and the occurrence of AAU but also a primary association with disease unrelated to linkage disequilibrium with other HLA class I or class II alleles. ${ }^{14}$ In particular, the disease association with HLA-Bw60 was shown to be secondary to linkage disequilibrium with LMP2B. Possible reasons for these discrepancies include clinical ascertainment error in so far as AAU may occur at any time during the course of AS, and the probable presence among different ethnic groups of different aetiological agents whose antigenic processing is variably influenced by the incorporation of LMP2 into the proteasome.

AS in Mexican Mestizo populations differs from AS in a white population in that juvenile onset of disease is far more common as is presentation with a characteristic enthesopathy and severe tarsal involvement. ${ }^{15-17}$ AAU, on the other hand, is far less common than AS in a white population. ${ }^{15}$ In view of data derived from Norwegian AS patients showing a preferential LMP2 gene disease association with 
Table 1 Spondyloarthropathy patient details of two populations of HLA-B27 positive Mexican Mestizo from Guadalajara and Mexico City

\begin{tabular}{lrrr}
\hline Patients & M:F & $\begin{array}{l}\text { Average age } \\
\text { (range) }\end{array}$ & $\begin{array}{l}\text { Age at onset } \\
\text { (range) }\end{array}$ \\
\hline $\begin{array}{l}\text { Guadalajara } \\
\text { Total patients }\end{array}$ & $68: 22$ & $34.4(16-59)$ & $23.4(6-46)$ \\
AAU+ & $9: 2$ & $40.9(21-54)$ & $24.7(6-46)$ \\
AAU- & $59: 20$ & $33.5(16-59)$ & $23.8(6-46)$ \\
PA+ & $45: 13$ & $32.7(16-56)$ & $20.6(6-46)$ \\
PA- & $23: 9$ & $37.7(21-59)$ & $27.4(12-46)$ \\
Mexico City & $70: 10$ & $24.1(11-55)$ & $16.1(5-52)$ \\
$\quad$ Total patients & $14: 1$ & $23.2(15-44)$ & $15.6(8-27)$ \\
AAU+ & $56: 9$ & $24.3(11-55)$ & $16.1(5-52)$ \\
AAU- & $17: 3$ & $22.9(14-55)$ & $16.9(8-52)$ \\
$\quad$ Undifferentiated spondyloarthropathy & & &
\end{tabular}

$\mathrm{AAU}=$ acute anterior uveitis, $\mathrm{PA}=$ peripheral arthritis. ${ }^{\star} 93.8 \%$ of Mexico City patients had peripheral arthritis. population also included 13 patients with juvenile onset (under 16 years) undifferentiated spondyloarthropathy (or seronegative enthesopathy and arthropathy (SEA) syndrome) ${ }^{20}$ and seven patients with adult onset undifferentiated spondyloarthropathy. Two patients with SEA syndrome had had AAU. AAU was confirmed by a complete ophthalmology assessment in both patient groups while peripheral arthritis was defined as arthritis occurring outside the axial skeletonthat is, excluding the shoulders and hips - and confirmed by a rheumatologist. Sacroiliitis was confirmed by the presence of a pelvic $x$ ray or computed tomography, or both. None of the patients had psoriasis or inflammatory bowel disease.

juvenile compared with adult onset disease, we have explored LMP2 gene disease associations in both juvenile and adult AS in a Mexican population in the hope that the findings may provide some clarification as to whether the primary influence of the LMP2 gene centres on the development of AAU or predisposes to a juvenile onset of disease. In a preliminary study of one Mexican population with AS, we were unable to discern any effect of LMP2 polymorphism on the phenotype of disease, although this study lacked sufficient numbers of AAU patients to arrive at any definitive conclusions. ${ }^{18}$ In this study, we have studied LMP2 gene polymorphism in two geographically distinct populations of Mexican Mestizo people with AS, one with predominantly juvenile onset and the other with predominantly adult onset disease, who have been well characterised clinically.

\section{Methods}

PATIENTS

AS was defined according to the modified New York criteria. ${ }^{19}$ Table 1 summarises patient details from two populations of Mexican Mestizo selected for HLA-B27 positivity. The Guadalajara study population consisted of 90 HLA-B27 positive Mexican Mestizo subjects with AS. Seventeen (18.9\%) of these had juvenile onset disease (that is, under 16 years of age). Eleven (12.2\%) patients had had AAU while $58(64.4 \%)$ had peripheral arthritis. The second population consisted of 80 HLA-B27 positive Mexican Mestizo subjects with AS from Mexico City. Fifty two $(65.0 \%)$ had juvenile onset disease (under 16 years of age). Fifteen $(18.8 \%)$ had had AAU and 75 (93.8\%) had peripheral arthritis. This Mexico City

Table 2 LMP2CfoI genotypes in HLA-B27 positive Mexican Mestizo spondyloarthropathy patients from Guadalajara and Mexico City

\begin{tabular}{llrr}
\hline & \multicolumn{3}{c}{ LMP2-CfoI genotype } \\
\cline { 2 - 4 } & $A A(\%)$ & $A B(\%)$ & \multicolumn{1}{c}{$B B(\%)$} \\
\hline Guadalajara & & & \\
$\quad$ Total patients & $4(4.4)$ & $23(25.6)$ & $63(70.0)$ \\
AS/AAU+ & $2(18.2)$ & $3(27.3)$ & $6(54.5)$ \\
Juvenile onset & $0(0)$ & $4(23.6)$ & $13(76.4)$ \\
$\quad$ AS/PA+ & $3(5.2)$ & $14(24.1)$ & $41(70.7)$ \\
Mexico City & & & \\
$\quad$ Total patients & $4(5.0)$ & $25(31.2)$ & $51(63.8)$ \\
AS/AAU+ & $2(13.3)$ & $6(40.0)$ & $7(46.7)$ \\
Juvenile onset & $2(3.8)$ & $18(34.6)$ & $32(61.6)$ \\
$\quad$ Undifferentiated & & & \\
$\quad$ spondyloarthropathy & $1(5.0)$ & $7(35.0)$ & $12(60.0)$ \\
\hline
\end{tabular}

\section{DNA EXTRACTION}

Genomic DNA was extracted from sodium EDTA anticoagulated blood using a modified salt precipitation method. ${ }^{21}$

\section{LMP2 GENE POLYMORPHISM}

LMP2 genotype assignments were made after polymerase chain reaction amplification of the second exon of LMP2 from genomic DNA followed by restriction enzyme digestion with Cfo1 as described previously. ${ }^{6}$ LMP2 genotype assignments were made before knowledge of the clinical history of each patient.

HLA TYPING

HLA-B27 typing was performed in the Department of Immunogenetics, INDRE, Mexico City, using standard microcytotoxicity assays and typing serum. Where this was not available (for the Guadalajara population), molecular typing for HLA-B27 was performed as described previously. ${ }^{22}$

\section{STATISTICAL ANALYSIS}

This was conducted mainly by stratified exact $2 \times 2$ and $2 \times 3$ table analysis. The predictor variable, the LMP2 allele, was categorised in two ways: first, the frequency and second, the prevalence of the LMP2A allele. The other predictor variable was population (Mexico City $v$ Guadalajara). To examine whether the LMP2 polymorphism primarily influences the development of AAU or predisposes to juvenile onset disease, we first considered age at onset as an outcome variable and tested LMP2A and B alleles, population (Mexico City $v$ Guadalajara), and development of AAU as predictor variables. Subsequently, development of AAU was considered as an outcome variable and LMP2A and B alleles, age at onset, and population as predictor variables. The EGRET epidemiological software was used to derive conditional maximum likelihood estimates (MLE) for the variable of interest. The stratified exact odds ratios (OR) are given together with $95 \%$ confidence intervals (CI).

\section{Results}

Table 2 illustrates the distribution of LMP2 genotypes in B27 positive subjects from Guadalajara and Mexico City subdivided according to presence or absence of AAU, peripheral 
Table 3 LMP2CfoI genotypes in Mexican AS patients stratified by age at onset and history of $A A U$

\begin{tabular}{clcr}
\hline \multicolumn{4}{c}{ LMP2-CfoI genotype } \\
\cline { 2 - 4 } & $A A(\%)$ & $A B(\%)$ & $B B(\%)$ \\
\hline $\begin{array}{c}\text { Guadalajara } \\
\text { Juveniles } \\
\text { AAU+ }\end{array}$ & 0 & $1(50)$ & $1(50)$ \\
AAU- & 0 & $3(20)$ & $12(80)$ \\
Adults & & & \\
AAU+ & $2(22.2)$ & $2(22.2)$ & $5(55.6)$ \\
AAU- & $2(3.1)$ & $17(26.6)$ & $45(70.3)$ \\
Mexico City† & & & \\
Juveniles & & & \\
AAU+ & $2(16.7)$ & $4(33.3)$ & $6(50)$ \\
AAU- & 0 & $14(35)$ & $26(65)$ \\
Adults & 0 & $2(66.7)$ & $1(33.3)$ \\
AAU+ & 0 & $5(20)$ & $18(72)$ \\
AAU- & $2(8.0)$ &
\end{tabular}

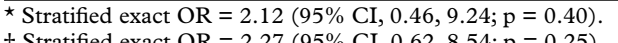
† Stratified exact OR $=2.27(95 \% \mathrm{CI}, 0.62,8.54 ; \mathrm{p}=0.25)$. Combined population stratified exact OR $=2.30$ (95\% CI, $0.90,5.91 ; \mathrm{p}=0.087)$. OR $=$ The exact conditional maximum likelihood estimates, stratified by age at onset of disease, for the odds ratio relating LMP2A allelic frequency and history of AAU.

Table 4 LMP2CfoI allelic frequencies in Mexican Mestizo with AS stratified by age at onset and history of $A A U$

\begin{tabular}{ccc}
\hline & \multicolumn{2}{l}{ LMP2-CfoI allele } \\
\cline { 2 - 3 } & A (\%) & $B(\%)$ \\
\hline $\begin{array}{l}\text { Guadalajara } \\
\text { Juveniles }\end{array}$ & \\
AAU+ & $1(25)$ & $3(75)$ \\
AAU- & $3(10)$ & $27(90)$ \\
Adults & & $12(66.7)$ \\
AAU+ & $6(33.3)$ & $107(83.6)$ \\
AAU- & $21(16.4)$ & \\
Mexico City & & $16(66.7)$ \\
Juveniles & $8(33.3)$ & $66(82.5)$ \\
AAU+ & $14(17.5)$ & $4(66.7)$ \\
AAU- & $2(33.3)$ & $41(82)$ \\
Adults & $9(18)$ & \\
AAU+ & & \\
AAU- & &
\end{tabular}

* Stratified exact $\mathrm{OR}=2.58(95 \% \mathrm{CI}, 0.80,7.62 ; \mathrm{p}=0.12)$. † Stratified exact OR $=2.31(95 \% \mathrm{CI}, 0.84,6.12 ; \mathrm{p}=0.11)$. Combined population stratified exact OR $=2.51$ (95\% CI, $1.21,5.09 ; \mathrm{p}=0.013)$. OR = The exact conditional maximum likelihood estimates, stratified by age at onset of disease, for the odds ratio relating LMP2A allelic frequency and history of AAU.

arthritis, and juvenile onset disease (under 16 years). The distribution of LMP2 genotypes in both populations of AS patients was similar. The prevalence of the LMP2 BB genotype was $(70 \%)$ and $(63.8 \%)$ in the Guadalajara and Mexico City populations, respectively.

Table 3 shows the LMP2 genotypes stratified according to age at onset and history of AAU. An increased prevalence of the LMP2A allele was noted in AAU positive versus AAU negative AS patients in both populations. Five of 11 AAU positive Guadalajara patients carried the LMP2A allele (45.4\%) compared with 22 of 79 AAU negative patients $(27.8 \%)$. Similarly, eight of 15 AAU positive Mexico City patients carried the LMP2A allele (53.3\%) compared with 21 of 65 AAU negative patients $(32.3 \%)$. The exact stratified by age at onset of disease conditional MLE for the odds ratios relating AAU and the prevalence of LMP2 A are provided in table 3. Exact odds ratios, stratified by history of AAU, with juvenile onset disease as the primary outcome showed no significant associations with LMP2A allelic prevalence (OR (Guadalajara)
$=0.67$, OR (Mexico City) = 1.19; OR (combined) $=1.07 ; \mathrm{p}>0.05$ for all analyses).

We have also analysed the prevalence of the LMP2 AA and LMP2AB genotypes and shown a significantly increased prevalence of the LMP2 AA genotype in AAU patients. The prevalence of LMP2 AA was $15.4 \%$ in AAU patients ( 4 of 26 ) versus $2.8 \%$ in non-AAU patients (4 of 144), while LMP2 AB was present in $34.6 \%$ (9 of 26) and $27.1 \%$ (39 of 144) of AAU and non-AAU patients, respectively. The exact $\mathrm{OR}$ stratified by age at onset relating AAU with the LMP2 AA and LMP2 AB genotypes were 9.03 (95\% CI, 1.42, 60.08; $\mathrm{p}=0.02)$ and 1.72 (95\% CI, 0.6, 4.78; $\mathrm{p}=0.36)$ respectively for the combined population. The few patients carrying genotype AA did not permit analysis for each city separately when stratified by age at onset of disease (table 3).

Table 4 shows that there was an increased allelic frequency for the LMP2A allele in AS patients who had had AAU compared with non-AAU patients in both Guadalajara and Mexico City. LMP2A allelic frequency in Guadalajara patients was $31.8 \%$ (7 of 22 alleles) in AAU positive subjects compared with $15.2 \%$ ( 24 of 158 alleles) in AAU negative subjects. LMP2A allelic frequency in Mexico City patients was $33.3 \%$ (10 of 30 alleles) in AAU positive subjects compared with $17.7 \%$ (23 of 130 alleles) in AAU negative subjects. The exact OR stratified by age at onset of disease are shown in table 4 . In neither population was LMP2A allelic frequency predictive of the development of juvenile onset disease when stratified by previous history of AAU $(\mathrm{OR}($ Guadalajara $)=0.59 ;$ OR (Mexico City) $=0.97 ; \mathrm{OR}$ (combined population) $=0.91 ; \mathrm{p}>$ 0.05 for all analyses).

The exact OR indicated no significant influence of either age at onset or disease population on the prevalence of AAU (data not shown). Within group comparisons of patients with and without peripheral arthritis showed no significant differences in the distribution of LMP2 genotypes or allelic frequencies ( $p>$ 0.05).

\section{Discussion}

Our study of two Mexican populations with spondyloarthropathy has provided consistent data to implicate polymorphism of the LMP2 locus in predisposition to AAU in HLA-B27 positive AS patients. LMP2 alleles did not seem to influence either age at onset or the development of peripheral arthritis.

At first hand, these findings seem to be at odds with previous studies in white populations of AS patients where the LMP2B rather than the LMP2A allele seems to predispose to AAU or juvenile onset disease, or both. ${ }^{5614}$ One explanation for these differences may reflect the distinct clinical background of spondyloarthritis in the Mexican Mestizo population. In addition to a much lower prevalence of AAU during the course of disease, juvenile onset is more common particularly in the setting of erosive disease, enthesopathy, and tarsal involvement compared with adult 
onset disease in white populations. Both our populations exemplified these clinical differences showing a low prevalence of AAU $(12.2 \%$ and $18.8 \%$ in the Guadalajara/Mexico City populations, respectively) and a high prevalence of peripheral arthritis $(64.4 \%$ and $93.8 \%$ of the Guadalajara/Mexico City populations, respectively). Patients with SEA were included with AS patients in the Mexico City cohort as follow up studies have shown that most patients presenting with SEA ultimately develop the more typical features of $\mathrm{AS}^{23}$

Although the explanation(s) accounting for this difference in the clinical spectrum of disease remains speculative, one might propose that different environmental agents (for example, bacteria) or immunopathogenetic mechanisms, or both, are responsible. Firstly, one might propose that the LMP2A allele influences the antigenic processing of a protein derived from one or more bacteria endemic to the Mexican population while the LMP2B allele conversely influences the processing of an endemic antigen to which white populations are exposed. Processing of a B27 binding motif that cross reacts with a uveitogenic cryptic self peptide(s) may stimulate autoreactive $\mathrm{T}$ cells demonstrating specificity for antigens in the anterior uvea. Secondly, recently described disease associations with other HLA alleles in Mexicans with spondyloarthropathy (for example, HLA-B49 and the FC31 complotype $)^{24}$ distinct from those seen in white populations (for example, HLA-Bw60) reinforces the concept of a distinct, multifactorial aetiology for this disease in different ethnic groups. Thus, competition between HLA-B alleles for binding of potential arthritogenic or uveitogenic peptides, or both, might influence the priming of autoreactive CTL. ${ }^{25}$ It seems unlikely, however, that differences in B27 subtypes from those seen in white patients with AS can acount for differences in disease phenotype as B27 subtyping in the Mexico City population (data not shown) as well as previous work $^{26}$ has shown that the predominant subtypes related to Mexican AS, B2702 and B2705, have the same prevalence as that seen in other AS populations. Further development of this hypothesis will require functional studies of LMP2 polymorphism in the context of potentially disease relevant antigens.

Our data do not preclude the possibility that the association of LMP2 polymorphism with predisposition to AAU simply reflects another disease locus in the HLA region on an extended HLA-B27 haplotype or on the non-B27 chromosome. Previous work in AS families raises the possibility of a second AS susceptibility gene on an extended B27/DR1 haplotype ${ }^{27}$ while other work has implicated HLA-Bw60. ${ }^{328}$ Consequently, the differing LMP2 allelic associations with disease in different populations may reflect differences in population prevalence of distinct disease associated HLA haplotypes.

The association between the presence of an $\mathrm{LMP} 2 \mathrm{~A}$ allele and predisposition to AAU is also consistent with the low prevalence of AAU in Mexican populations with spondyloarthritis. Thus, the A allele is much less common than the $\mathrm{B}$ allele in Mexican patients with spondyloarthritis $(18.8 \% \quad v \quad 81.2 \%)$ while homozygosity for the A allele was seen in only $4.4 \%$ and $5.0 \%$ of the Guadalajara/Mexico City AS populations, respectively. In contrast, $50 \%$ of all LMP2 AA homozygotes had had AAU. Consequently, one explanation for the low prevalence of AAU in Mexican AS might be the requirement for both an LMP2A allele and HLA B27 in the processing and presentation of a disease relevant antigen endemic to Mexico that is associated with the development of AAU.

In conclusion, our findings in two Mexican populations of $\mathrm{B} 27$ positive patients with juvenile and adult onset AS support the view that polymorphism of the LMP2 gene is associated with the development of AAU rather than age at onset. Our results also suggest potential immunopathological mechanisms that might account for different clinical presentations of spondyloarthropathy in different parts of the world.

Walter P Maksymowych is a scholar with the Alberta Heritage Foundation for Medical Research. This work was supported by an operating grant from the Medical Research Council of an operatin

1 van der Linden SM, Valkenburg HA, de Jongh B, Cats A. The risk of developing ankylosing spondylitis in HLA-B27 positive individuals: a comparison of relatives of spondylitis positive individuals: a comparison of relatives of spondylitis
patients with the general population. Arthritis Rheum patients with

2 Calin A, Marder A, Becks E, Burns T. Genetic differences between B27-positive patients with ankylosing spondylitis and B27-positive healthy controls. Arthritis Rheum 1983; 26:1460-4.

3 Robinson WP, van der Linden SM, Khan MA, Rentsch HU, Cats A, Russell A, et al. HLA-Bw60 increases susceptibility to ankylosing spondylitis in HLA-B27+ patients. Arthritis Rheum 1989;32:1135-41.

4 Monaco JJ, McDevitt HO. H2-linked low-molecular-weight polypeptide antigens assemble into an unusual macromolecular complex. Nature 1984;309:797-9.

5 Maksymowych WP, Wessler A, Schmitt-Egenolf M, SuarezAlmazor M, Ritzel G, Von Borstel RC, et al. Polymorphism in an HLA-linked proteasome gene influences phenotypic expression of disease in HLA-B27 individuals. J Rheumatol 1994;21:665-9.

6 Maksymowych WP, Suarez-Almazor M, Chou CT, Russell AS. Polymorphism in the LMP2 gene influences susceptibility to extraspinal disease in HLA-B27 positive individuals with ankylosing spondylitis. Ann Rheum Dis 1995; 54:321-4.

7 Goldberg AL, Rock K. Proteolysis, proteasomes, and antigen presentation. Nature 1992;357:375-9.

8 Aki M, Shimbara N, Takashina M, Akiyama K, Kagawa S, Tamura $\mathrm{T}$, et al. Interferon- induces different subunit organizations and functional diversity of proteasomes. J Biochem 1994;115:257-69.

9 Früh K, Gossen M, Wang K, Bujard H, Peterson PA, Yang Y. Displacement of housekeeping proteasome subunits by modulating the multicatalytic proteinase complex. EMBO J 1994;13:3236-44.

10 Van Kaer L, Ashton-Rickardt PG, Eichelberger M, Gaczynska M, Nagashima K, Rock KL, et al. Altered
peptidase and viral-specific $T$ cell response in LMP2 peptidase and viral-specific $T$ cell respo
mutant mice. Immunity $1994 ; 1: 533-41$.

11 Fehling HJ, Swat W, Laplace C, Khun R, Rajewsky K, Muller U, et al. MHC class I expression in mice lacking the proteasome subunit LMP-7. Science 1994;265:1234-7.

12 Glynne R, Powis SH, Beck S, Kelly A, Kerr L, Trowsdale J. A proteasome-related gene between the two ABC transporter loci in the class II region of the human MHC. Nature 1991;353:357-60.

13 Burney RO, Pile KD, Gibson K, Calin A, Kennedy LG, Sinnott PJ, et al. Analysis of the MHC class II encoded components of the HLA class I antigen processing pathway in ankylosing spondylitis. Ann Rheum Dis 1994; 53:58-60.

14 Ploski R, Forre O, Flato B, Vinje O, Maksymowych W, Thorsby E. Association to HLA-DRB ${ }^{\star} 08$, HLADPB $1^{\star} 03$ and homozygosity for an HLA-linked proteaDPB ${ }^{\star} 03$ and homozygosity for an HLA-linked protea-
some gene in juvenile ankylosing spondylitis. Human some gene in juvenile ant
Immunol $1995 ; 44: 88-96$. 
15 Burgos-Vargas R, and Vázquez-Mellado J. The early clinical recognition of juvenile-onset ankylosing spondylitis and its differentation from juven

16 Burgos-Vargas, Naranjo A, Castillo J, Katona G. Ankylosing spondylitis in the Mexican Mestizo: patterns of disease according to age at onset. J Rheumatol 1989;16:186-91

17 Ramos-Remus C, Gomez-Vargas A, Gamez-Nava JI, Gonzalez-Lopez L, Maksymowych WP, Farrera-Gamboa $\mathrm{J}$, et al. Neurologic involvement in patients with ankylosing spondylitis (AS) with atlantoaxial subluxation (AAS): A clinical and neurophysiological study. J Rheumatol 1995; 22:2120-5.

18 Maksymowych WP, Russell AS, Ramos-Remus C, SanchesCorona J. Polymorphism in the LMP2 gene does not influence disease phenotype in Mexican individuals with ankylosing spondylitis. Arthritis Rheum 1994;37 (suppl):250.

19 van der Linden S, Valkenburg HA, Cats A. Evaluation of diagnostic criteria for ankylosing spondylitis: a proposal for modification of the New York criteria. Arthritis Rheum 1984;27:361-8

20 Zeidler H, Wilfried M, Khan MA. Undifferentiated spondyloarthropathies. Rheum Dis Clin North Am 1992;18:187202 .

21 Gustincich S, Manfioletti G, Del Sal G, Schneider C. A fast method for high-quality genomic DNA extraction from whole human blood. Biotechniques 1991;11:298-302.
22 Dominguez O, Coto E, Martinez-Naves E, Choo SY, Lopez-Larrea C. Molecular typing of HLA-B27 alleles. Lopez-Larrea C. Molecular typin
Immunogenetics 1992;36:277-82.

23 Burgos-Vargas R, Clark P. Axial involvement in the seronegative enthesopathy and arthropathy syndrome and its progression to ankylosing spondylitis. J Rheumatol 1989;16:192-7.

24 Vargas-Alarcón G, Garcia A, Bahena S, Melin-Aldana $\mathrm{H}$, Andrade F, Ibanez-de-Kasep G, et al. HLA-B alleles and
complotypes in Mexican patients with seronegative spondyloarthropathies. Ann Rheum Dis 1994;53:755-8.

25 Tussey LG, Rowland-Jones S, Zheng TS, Androlewicz MJ, Cresswell P, Frelinger JA, et al. Different MHC Class I alleles compete for presentation of overlapping viral epitopes. Immunity 1995;3:65-77.

26 López-Larrea C, Gonzalez-Roces S, Peña M, Dominguez $\mathrm{O}$, Coto E, Alvarez V, et al. Characterization of B27 haplotypes by oligotyping and genomic sequencing in the Mexican Mestizo population with ankylosing spondylitis: juvenile and adult onset. Human Immunol 1995;43;174-80.

27 Brown MA, Pile KD, Klinke J, Gibson K, Bunce M, Calin A, et al. HLA class II associations of ankylosing spondyliA, et al. HLA class II associations of
tis. Ann Rheum Dis 1996;55:584.

28 Brown MA, Pile KD, Kennedy LG, Calin A, Darke C, Bell $\mathrm{J}$, et al. HLA class I associations of ankylosing spondylitis in the white population of the United Kingdom. Ann Rheum Dis 1996;55:268-70. 